\title{
Efeitos de Conteúdos Apreciativos e Depreciativos de Premissas no Raciocínio Dedutivo de Estudantes de Psicologia
}

\author{
Simone Cagnin ${ }^{1, *}$ \\ Orcid.org/0000-0002-8977-3290 \\ Maria Isabel da Silva Leme ${ }^{2}$ \\ Orcid.org/0000-0003-0844-3554 \\ ${ }^{1}$ Universidade do Estado do Rio de Janeiro, Rio de Janeiro, RJ, Brasil \\ ${ }^{2}$ Universidade de São Paulo, São Paulo, SP, Brasil
}

\section{Resumo}

Estudos recentes na Psicologia Cognitiva apresentam evidências da influência dos conteúdos afetivos contidos em enunciados de problemas no desempenho em tarefas de raciocínio dedutivo. Este estudo investiga a influência de conteúdos valorativos relacionados à formação e à atuação profissional dos psicólogos no desempenho de estudantes de Psicologia em tarefas que envolvem silogismos categóricos. Visa ainda investigar se a integração acadêmica desses estudantes, avaliada através do "Questionário de Vivências Acadêmicas reduzido" (QVA-r), teve relação com a sensibilidade a estes conteúdos nas tarefas lógicas. Participaram da pesquisa 480 estudantes de cursos de Psicologia de universidades públicas brasileiras, sendo estes divididos, aleatoriamente, em três condições de conteúdo valorativo: neutro, apreciativo e depreciativo. Os resultados obtidos sugerem um efeito negativo dos conteúdos depreciativos no desempenho das estudantes do sexo feminino. Não foram encontradas relações relevantes entre as dimensões da integração acadêmica (pessoal, interpessoal, carreira, estudo e institucional) e o número de respostas válidas nos silogismos, o que aponta para um efeito per se desses conteúdos. Acredita-se que os resultados possam ter relevância para o contexto acadêmico, pois as situações-problema vivenciadas pelos alunos na universidade podem revestir-se de conteúdos valorativos que podem ter influência no raciocínio dedutivo, bem como em outros processos de diferentes naturezas.

Palavras-chave: Raciocínio, resolução de problemas, conteúdos afetivos.

\section{The Effects of the Evaluative Content of Premises on the Deductive Reasoning of Psychology Students}

\begin{abstract}
Recent studies in cognitive psychology show evidence of the influence of affective content in statements relating to performance in deductive reasoning tasks. This study investigates the influence of evaluative

* Endereço para correspondência: Av. São Francisco Xavier, 524, 10 andar, salas 10022/ 10028, Maracanã, Rio de Janeiro, RJ, Brasil 20550-900. Fone: 2334 0234. E-mail: simcagnin@gmail.com

Agradecimento: As autoras gostariam de agradecer ao professor da Universidade do Estado do Rio de Janeiro, José Augusto Evangelho Hernandez, pela assessoria na análise dos dados.
\end{abstract}


content related to the education and professional practice of psychologists on the performance of psychology students in tasks involving categorical syllogisms. It also aims to investigate if there is a relationship between the academic integration of these students, who were evaluated by means of the "Questionário de Vivências Acadêmicas reduzido" (QVA-r), and their sensitivity to this content in logical tasks. Participants were 480 psychology students from Brazilian public universities. They were randomly assigned three evaluative content situations: neutral, appreciative and depreciative. Results suggest that depreciative content has a negative effect on the performance of female students. No relevant relationships were found between academic integration (personal, interpersonal, career, studies and institutional) and the number of valid replies to the syllogisms, which points to the effect per se of this content. The results are relevant in an academic context, since the problem-situations experienced by college students may contain evaluative content that can have an influence on deductive reasoning and other processes of a different nature.

Keywords: Reasoning, problem-solving, affective content.

\section{Efectos de Contenido Evaluativo de las Premisas en el Razonamiento Deductivo de los Estudiantes de Psicología}

\section{Resumen}

Estudios recientes en la Psicología Cognitiva han mostrado evidencia de la influencia del contenido afectivo que recubre los problemas de razonamiento lógico. Este estudio tiene como objetivo investigar la influencia de contenido evaluativo sobre la formación y sobre la práctica profesional de psicólogos en el rendimiento de los estudiantes de psicología en las tareas lógicas que implican silogismos categóricos. También busca investigar si la integración académica de los estudiantes, determinada por el "Questionário de Vivências Acadêmicas reduzido" (QVA-r), estaba relacionado con la sensibilidad a estos contenidos en tareas lógicas. 480 estudiantes de Psicología de universidades públicas brasileñas participaron del estudio y fueron divididos aleatoriamente en tres condiciones de evaluación, contenido neutral, apreciativo y despectivo. Los resultados sugieren un efecto negativo de los contenidos despectivos en las mujeres. No hubo relaciones significativas entre las dimensiones de la integración académica y las respuestas válidas en los silogismos, lo que apunta a un efecto per se de contenido evaluativo. Los hallazgos podrían tener especial importancia para el contexto escolar, en la medida en que las situaciones problemáticas enfrentadas por los estudiantes universitarios en su rutina academica pueden ser de contenido evaluativo que pueden influenciar el desempeño en el razonamiento deductivo $\mathrm{y}$ en otros procesos de naturalezas diferentes.

Palabras clave: Razonamiento, resolución de problemas, contenidos afectivos.

Evidências obtidas em estudos sobre o raciocínio humano sugerem que os conteúdos das proposições podem influenciar, de modo significativo, como as pessoas raciocinam como, por exemplo, quando a tarefa envolve conteúdos familiares. Esses estudos evidenciam que, não só o raciocínio indutivo, mas também o raciocínio hipotético-dedutivo, estão sujeitos à influência dos conteúdos das proposições e premissas (Tversky \& Kahneman, 1983). E ainda, frequentemente, as deduções lógicas avaliam as consequências da aplicação de regras, leis e princípios morais, e esta habilidade pode variar nos indivíduos em função da inteligência e da capacidade da Memória de Trabalho (Johnson-Laird, Khemlani, \& Goodwin, 2015).

Observa-se que, apesar da multiplicidade de linhas de pesquisas encontradas na área, um número expressivo destas investigações está baseado na abordagem do processamento da informação, investigando a influência de conteúdos de enunciados semânticos neutros na resolução 
de problemas de diferentes naturezas, incluindo-se problemas lógicos que envolvem raciocínio dedutivo, ou seja, de conteúdos que não apresentam tonalidade afetiva nos enunciados dos problemas (e.g. De Neys, 2012). Lefford (1946) foi um dos primeiros pesquisadores que comprovou que os indivíduos ao resolver silogismos com conteúdos afetivos negativos cometiam mais erros lógicos do que com neutros, o que foi confirmado em estudos posteriores (Blanchette \& Leese, 2011; Jung, Wranke, Hamburger, \& Knauff, 2014).

Um número crescente de estudos (De Jong, \& Vroling, 2014; De Neys, 2012, 2014) vem apontando para a influência das crenças e expectativas dos indivíduos no raciocínio dedutivo humano. Os achados de muitos desses estudos têm demonstrado que quando os conteúdos dos silogismos e das tarefas lógicas coincidem com as crenças e o conhecimento cotidiano dos indivíduos, há melhora no desempenho nessas tarefas. Em contraste, quando ocorre conflito entre as crenças e expectativas e a lógica, o desempenho costuma ser negativamente afetado, especialmente nas formas logicamente inválidas de silogismos, ou seja, na "Afirmação do Consequente" e na "Negação do Antecedente". Observam-se também estudos que enfatizam a influência dos estados afetivos no raciocínio, incluindo-se aqui estados de humor eliciados por estímulos experimentais e/ou emoções mais específicas, bem como a influência per se da tonalidade dos conteúdos dos enunciados de problemas que envolvem raciocínio dedutivo (Blanchette \& Campbell, 2012; Blanchette \& Leese, 2011; Blanchette \& Richards, 2010). Há, nestas pesquisas, muitas vezes, a manipulação dos conteúdos dos enunciados das premissas de acordo com a valência do estado afetivo eliciado nos indivíduos (positiva, negativa ou neutra). Neste contexto, costumam ser também avaliados, os efeitos da congruência ou incongruência entre estados afetivos negativos e positivos e enunciados e premissas com conteúdos negativos e positivos, onde a similaridade de valência, muitas vezes, facilita o desempenho.

Apesar de evidências que relatam o efeito negativo de estados e de conteúdos afetivos negativos no raciocínio dedutivo (Blanchette \& Leese, 2011; Jung et al., 2014; Lefford, 1946), há também estudos que indicam que estados afetivos positivos podem influenciar negativamente o desempenho em tarefas lógicas que envolvem silogismos (Melton, 1995), pela sua interferência no processamento mais analítico requerido pela aplicação de regras lógicas. Por outro lado, há estudos que apontam para efeitos benéficos de estados e de conteúdos afetivos positivos em determinados tipos de tarefas formais, como as que envolvem silogismos, ou informais de raciocínio (Blanchette \& Caparos, 2013; Eliades, Mansell, Stewart, \& Blanchette, 2012).

Esses resultados questionam a ideia prevalente de que os conteúdos afetivos negativos têm sempre uma influência negativa no desempenho em tarefas lógicas. Como destacam os autores acima, em algumas circunstâncias, esses conteúdos podem favorecer o desempenho lógico, especialmente quando há compatibilidade entre a valência da tonalidade afetiva dos conteúdos com a valência do estado afetivo do indivíduo. Isso porque como apontam os estudos de Blanchette e Caparos (2013) e Blanchette, Lindsay e Davies (2014), a relevância e a utilidade dos conteúdos, ainda que negativos, podem favorecer o desempenho de indivíduos que se sentem identificados com o teor desses conteúdos.

Como postula um dos modelos mais utilizados para explicar o impacto dos conteúdos afetivos sobre o raciocínio, existem dois sistemas que podem ser recrutados em tarefas que demandam raciocínio lógico, como propôs Kahneman (2003), utilizando, por sua vez, os termos "Sistema 1" e "Sistema 2 mencionados inicialmente por Stanovich e West (2002). O chamado "Sistema 1" envolve processos heurísticos, implícitos, rápidos, amplamente contextualizados e baseados em conhecimento prévio. O chamado "Sistema 2" envolve processos analíticos, explícitos, lentos, baseados em regras, que requerem a alocação de recursos cognitivos e sobrecarga na Memória de Trabalho. Diferentes estudos como os de Blanchette e Amato (2014) apontam para a dominância de processos heurísticos, implícitos, em situações-problema onde há o confronto entre emoção e razão, com a consequente diminuição 
de um processamento mais analítico e explícito requerido por tarefas lógicas que demandam, por um lado, atenção e sobrecarga cognitiva, por outro, a contenção de respostas automáticas e de interferência de crenças e conhecimentos dos solucionadores. Nesta perspectiva, as pessoas em geral tendem a tecer julgamentos e a raciocinar logicamente a partir de impressões rápidas, intuitivas, mais do que de modo deliberado e analítico (Evans, 2008; Kahneman, 2003; Kahneman \& Frederick, 2005).

Blanchette (2014) observa que muitos resultados de estudos são consistentes com a literatura sobre atenção e memória, pois as informações de cunho afetivo atraem mais atenção, e as informações congruentes com o estado afetivo se tornam mais acessíveis na Memória de Longo-Prazo. Segundo essa autora, a emoção fornece informações relevantes a serem aplicadas nas tarefas de raciocínio, incluindo-se o raciocínio dedutivo, alterando como a informação é processada e influenciando qual informação adicional pode ser usada nestas tarefas. Nesta perspectiva, para Blanchette e Richards (2010), os estados afetivos podem mudar o contexto semântico construído nos processos de inferência, potencializando os efeitos da afetividade no raciocínio dedutivo, o que parece corroborar a hipótese de que a afetividade pode servir como "fonte de informação" e poderia explicar, em parte, como os estados afetivos afetam o raciocínio dedutivo. Não obstante, esse priming semântico pode variar dependendo de fatores contextuais como a natureza da tarefa e a complexidade da informação. Porém, os processos afetivos vão além de um processamento semântico, pois envolvem processos não informacionais qualitativamente diferentes, ou seja, processos não cognitivos, ou como preferem Nicolle e Goel (2014), processos diretamente ligados à "emoção como emoção". Dentre os aspectos não informacionais das emoções, encontram-se as alterações fisiológicas e, segundo Blanchette (2014), esta seria a segunda rota através da qual a afetividade afeta o raciocínio, incluindo-se aqui o raciocínio dedutivo.

A percepção do conflito entre a lógica e a crença costuma ser implícita e muitas vezes avaliada através de medidas relacionadas aos aspectos não informacionais da emoção como os acima referidos. Uma espécie de "sentimento lógico" difuso que se expressa, nos indivíduos, por uma sensação de "incômodo" e de incerteza quanto às suas respostas parece surgir quando há conflito entre as crenças e a validade lógica da tarefa. Inclusive, Boden e Barenbaum (2010) destacam a relação estreita entre emoções e crenças, propondo uma espécie de "alça de feedback" entre elas. Quando, em uma situação-problema, há incerteza e ambiguidade, aumenta a chance da interferência de crenças e expectativas pessoais no lugar de conhecimentos abstratos e regras lógicas na resolução.

Observa-se ainda, em alguns estudos na área, a apresentação de histórias com proposições com conteúdos afetivos ou neutros antes das tarefas que envolvem a resolução de silogismos com conteúdos afetivos ou neutros (Gangemi, Mancini, \& Johnson-Laird, 2013). Em outros estudos, os participantes são instruídos a responder a perguntas sobre as emoções vivenciadas em uma situação traumática (e.g. frente a um ataque terrorista) antes das tarefas com silogismos propriamente ditas (Blanchette, Richards, Melnyk, \& Lavda, 2007). Assim, o uso de histórias prévias aos silogismos ou a instrução para a lembrança de vivências relacionadas a situações traumáticas antes da apresentação de silogismos parecem "potencializar" os efeitos dos conteúdos afetivos dos últimos, ao favorecer um contexto de mesma tonalidade afetiva. Com o objetivo de "potencializar" possíveis efeitos de priming afetivo, no estudo aqui apresentado também foram apresentadas histórias prévias às tarefas com silogismos que, por sua vez, versam sobre a formação e atuação profissional dos psicólogos, nos grupos de conteúdos valorativos, e sobre a distribuição dos cursos de psicologia no Brasil, no grupo de conteúdo neutro.

Quanto aos efeitos diferenciados de variáveis como o sexo e as vivências sobre o desempenho em tarefas de raciocínio com conteúdos afetivos, Brody e Hall (2008) ressaltam que as pesquisas que focalizam as diferenças entre homens e mulheres verificaram que essas são mediadas por variáveis sócio culturais, cognitivas, biológicas, e comportamentais. Costuma 
ser recorrente o estereótipo de que as mulheres são mais emocionais do que os homens, expressando e verbalizando mais emoções do que estes, porém dados empíricos sugerem uma maior complexidade associada à esta questão. No que se refere à regulação das emoções, alguns estudos observam que as mulheres tendem a usar, de modo mais prevalente, estratégias interpessoais, bem como estratégias de ruminação que focalizam mais a atenção em afetos negativos (Nolen-Hoeksema \& Jackson, 2001), o que poderia explicar, em parte, uma maior sensibilidade do sexo feminino aos conteúdos afetivos negativos. Porém, aspectos de personalidade e o grau de motivação podem dificultar ou facilitar essa regulação, somando-se assim a possíveis efeitos de diferenças entre os sexos. Em função destes resultados, uma das hipóteses da presente pesquisa é a de que as estudantes do sexo feminino apresentarão maior sensibilidade a estes conteúdos, em especial aos conteúdos valorativos depreciativos. O presente estudo visa esclarecer, em parte, estes efeitos tendo em vista os resultados sugestivos encontrados em estudos preliminares, onde as mulheres apresentaram mais erros lógicos frente a este tipo de conteúdo, com desempenho equivalente ao dos homens frente aos conteúdos neutros.

Por fim, cabe analisar ainda a importância de vivências como a integração acadêmica dos participantes e de sua possível relação com o desempenho dos mesmos nas tarefas com silogismos categóricos que fazem apreciações ou depreciações sobre a sua formação e a atuação profissional. Pesquisas contemporâneas têm buscado investigar a satisfação e/ou as vivências acadêmicas em estudantes universitários (Granado, Santos, Almeida, Soares, \& Guisande, 2005; Igue, Bariani, \& Milanesi, 2008; Lamas, Ambiel, \& Silva, 2014; Sarriera, Paradiso, Shutz, \& Howes, 2012; Schleigh, 2006; Schleigh, Polydoro, \& Santos, 2006; Soares et al., 2014; Tessema, Ready, \& Yu, 2012). Inclusive, alguns estudos (Igue et al., 2008; Lamas et al., 2014; Sarriera et al., 2012; Schleigh, 2006) utilizaram o QVA-r visando avaliar, neste contexto, a integração acadêmica de universitários, muitas vezes, relacionando-a com outros aspectos da vida universitária e/ou da profissão, dentre outros aspectos. E, como sinaliza Granado (2004), o constructo 'integração universitária' é um fenômeno multifacetado, que integra a influência de características pessoais, expectativas, habilidades, com a influência de características institucionais, contextuais e as interações que ocorrem na universidade.

Os resultados desses estudos, em geral, indicam que a dimensão carreira em comparação com as dimensões pessoal, interpessoal, estudo e institucional, que compõem os 55 itens do QVA-r, apresenta médias mais elevadas que as demais, o que sugere que os universitários parecem possuir, em geral, uma percepção positiva em relação à escolha do curso e às perspectivas profissionais. Já a média mais baixa normalmente encontrada na dimensão pessoal poderia indicar uma percepção mais negativa de aspectos relacionados ao bem-estar físico e psicológico, o que poderia sugerir uma percepção de si como tendo menor estabilidade afetiva e baixo nível de confiança e auto conceito (Schleigh, 2006).

Cabe assinalar que a aplicação do QVA-r, em nosso estudo, objetivou esclarecer, por um lado, possíveis relações entre aspectos subjetivos de estudantes universitários, tais como ansiedade, variações de humor, expectativa profissional e engajamento no curso escolhido, com a maior sensibilidade aos conteúdos valorativos de silogismos relacionados à formação e atuação profissional dos mesmos. Assim, o nosso objetivo não foi o de investigar a integração acadêmica per se, como propõem os estudos acima mencionados, mas sim o de explorar se há relações entre a integração acadêmica e o desempenho nas tarefas lógicas que envolvem silogismos com conteúdos relacionados à formação e à atuação profissional dos estudantes.

Em suma, o estudo visa investigar a influência de conteúdos valorativos de silogismos no desempenho de estudantes universitários, conteúdos estes que apresentam juízos de valor, positivos ou negativos, em relação à futura profissão desses estudantes e, ainda, se estes se relacionam com expectativas e crenças em relação ao seu curso e profissão, reforçando o impacto que os mesmos possam vir a ter nesse desempenho. 
Diferenças de desempenho entre os sexos serão aqui consideradas, bem como possíveis relações entre a integração acadêmica dos participantes e uma maior sensibilidade a estes conteúdos.

\section{Método}

A pesquisa aqui relatada é composta por dois estudos experimentais transversais complementares com design: 3 (grupos) x 2 (sexos) e 3 (grupos) x 4 (tipos de silogismos), respectivamente, sendo as variáveis dependentes, o desempenho nas tarefas com os silogismos e nos questionários aplicados. Foram também exploradas as relações entre o desempenho nas tarefas com os silogismos e a integração acadêmica e suas dimensões como medidas pelo QVA-r.

\section{Participantes}

A amostra final de conveniência foi constituída por 480 estudantes universitários (318 do sexo feminino e 162 do sexo masculino), com média de idade de 22,4 anos $(D P=5,44)$, variando de 17 a 49 anos, de cursos de Psicologia de sete universidades públicas brasileiras localizadas em seis estados e três regiões brasileiras (sudeste, sul e nordeste). Não houve diferença significativa na distribuição dos participantes nos grupos $\left[X^{2}(2)=0,042 ; p>0,05\right]$ e não houve diferença significativa entre as médias das respostas nas tarefas dos silogismos por região $[F$ $\left.(2,477)=2,77 ; p>0,05 ; \boldsymbol{\eta}^{2}=0,12\right]$.

Com o intuito de equiparar a amostra, foram excluídos, de modo aleatório, mas mantendo a proporcionalidade em cada grupo, 31 estudantes do sexo feminino. Foram também excluídos 8 participantes, 7 mulheres e um homem, que deixaram pelo menos uma resposta em branco no QVA-r e/ou na tarefa com silogismos, conforme critério previamente estabelecido. Não houve diferença significativa $[(t$ (509) $=0,01 ; p>0,05$; $d=0,007]$ entre a média das participantes aleatoriamente excluídas $(M=6,41 ; D P=1,47)$ e a da amostra final $(M=6,42 ; D P=1,42)$ no desempenho nas tarefas com silogismos, bem como não houve diferença significativa entre as médias $(M=3,62 ; D P=0,32 ; M=3,53 ; D P=0,42$, respec- tivamente) nas respostas no QVA-r $[t(509)=1,2$; $p>0,05 ; d=0,24]$.

\section{Instrumentos}

No que tange à avaliação dos efeitos dos conteúdos valorativos no raciocínio silogístico dos estudantes, objetivo desse estudo, foram construídos 8 silogismos, sendo dois em cada forma de silogismo (Modus Ponens, Modus Tollens, Afirmação do Consequente e Negação do Antecedente). Desses oito silogismos, quatro continham premissas com nomes femininos e quatro com nomes masculinos. Um exemplo de silogismo apresentado "Todo psicólogo valoriza a ética em sua atuação. Joaquim não valoriza a ética em sua atuação. Logo Joaquim não é psicólogo". Os enunciados dos silogismos e as histórias que os antecederam foram construídos procurando-se equiparar as palavras em comum entre eles, o tamanho das frases, a contraposição de ideias tendo em vista os adjetivos usados e as apreciações e depreciações feitas. Neste contexto, o grau de dificuldade da tarefa foi também equiparado.

Antes da apresentação da tarefa com os silogismos foram apresentadas histórias que versaram sobre a percentagem e a distribuição de cursos de Psicologia no Brasil (história neutra, apresentada ao grupo de conteúdos neutros) ou sobre a formação e a atuação do psicólogo. Nesse último caso, as histórias valorizavam esta formação e atuação, nos grupos de conteúdos apreciativos, ou depreciavam essa formação e atuação, nos grupos de conteúdos depreciativos. O objetivo da apresentação de histórias antes dos silogismos foi o de potencializar os efeitos da tonalidade valorativa dos conteúdos das premissas.

Foram também aplicados, nos grupos, quatro questões avaliadas por meio de escalas tipo Likert de 4 pontos, graduadas a partir da opção "nenhuma" à opção "muita", para obter informações, respectivamente, sobre a experiência dos estudantes na resolução de silogismos, sobre o grau de dificuldade encontrado na realização das tarefas, a respeito da concordância/discordância dos participantes em relação às avaliações con- 
tidas nos enunciados, e se estes se sentiram envolvidos afetivamente pelo teor dos enunciados. Foi indagada, em uma questão aberta e opcional, a opinião dos participantes sobre a tarefa.

Outro instrumento utilizado foi o Questionário de Vivências Acadêmicas, (QVA-r), versão reduzida, apresentado a todos os participantes antes da leitura das histórias e da tarefa com silogismos. A aplicação desse questionário objetivou investigar se a integração acadêmica dos universitários teria relação com as respostas válidas nas tarefas com silogismos. Este questionário tem sua origem na versão portuguesa construída e validada por Almeida, Soares e Ferreira (2001), adaptada e validada para o contexto brasileiro por Granado et al. (2005); Santos, Noronha, Amaro e Villar (2005), apresentando qualidade psicométrica significativa, em especial, com boa consistência interna ( $\boldsymbol{\alpha}=$ $0,88)$, mostrando-se adequado para ser usado no contexto brasileiro. O QVA-r é um instrumento de auto relato organizado como uma escala tipo Likert de 5 pontos ( $1=$ nada a ver comigo até $5=$ tudo a ver comigo). A versão brasileira apresenta 55 itens divididos em 5 dimensões, pessoal (14 itens), interpessoal (12 itens), carreira (12 itens), estudo (9 itens) e institucional (8 itens). A dimensão pessoal avalia a percepção de bem-estar psicológico e físico $(\boldsymbol{\alpha}=0,84)$; a dimensão interpessoal $(\boldsymbol{\alpha}=0,82)$, o relacionamento com os colegas e a estabilidade destas relações. A dimensão carreira $(\boldsymbol{\alpha}=0,86)$ avalia a percepção de competências pessoais associadas à carreira escolhida e às perspectivas profissionais, e a dimensão estudo $(\boldsymbol{\alpha}=0,78)$ avalia rotinas de estudo, o planejamento e a execução das tarefas escolares. A dimensão institucional $(\boldsymbol{\alpha}=0,77)$ avalia a instituição de ensino, sua infraestrutura e serviços (Granado, 2004). Uma questão aberta sobre comentários sobre a vida universitária foi feita após a apresentação do QVA-r.

\section{Procedimento}

Após a aprovação do Comitê de Ética (parecer 015-2013), foi solicitada permissão para a realização da pesquisa junto às universidades públicas e aos professores. Em seguida, foi soli- citada a participação dos estudantes que responderam individualmente aos materiais aplicados em suas salas de aula. A participação foi voluntária e o Termo de Consentimento Livre e Esclarecido foi distribuído e assinado por todos, com a garantia da observância dos procedimentos éticos de pesquisa.

Os participantes responderam individualmente aos materiais descritos acima na seguinte ordem: Questionário de Vivências Acadêmicas, leitura de histórias, tarefa com silogismos e questionários sobre a tarefa.

Inicialmente, após o preenchimento da folha de rosto com informações sobre o sexo, idade, período e universidade, foi apresentado aos participantes o Questionário de Vivências Acadêmicas (QVA-r), seguido de uma questão aberta e opcional sobre a opinião dos mesmos sobre a vida universitária. A seguir, foram apresentadas histórias de conteúdos afetivos neutros, apreciativos e depreciativos, para os grupos de conteúdos neutros, apreciativos e depreciativos, respectivamente. Após a leitura das histórias, foram apresentadas as tarefas com os quatro tipos de silogismos (Modus Ponens, Modus Tollens, Afirmação do Consequente e Negação do Antecedente), de maneira randômica, em cada turma, para três grupos de estudantes distintos (tarefas com silogismos com conteúdos neutros, apreciativos e depreciativos). Após a leitura de cada silogismo, foi pedido que os participantes assinalassem se as conclusões seriam ou não logicamente derivadas das afirmações feitas na primeira premissa. Por fim, foram apresentadas quatro breves questões que versavam sobre: a opinião sobre a dificuldade da tarefa, a experiência com silogismos, a concordância ou discordância com o teor dos silogismos apresentados, o envolvimento afetivo com o teor dos enunciados da tarefa.

A aplicação durou, em média, 30 minutos e, no final, foi feito um agradecimento pela participação e assumido o compromisso da divulgação dos resultados.

Cabe observar que os materiais foram pré-testados com 30 participantes, para ajustar, se fosse o caso, os instrumentos e procedimentos, especialmente no que se refere à opinião dos 
mesmos sobre os conteúdos afetivos valorativos das histórias e sobre a compreensão e relevância da tarefa com silogismos. Houve consenso, em um questionário aberto aplicado, sobre o teor dos conteúdos afetivos dos silogismos e das histórias (neutros, apreciativos e depreciativos) e sobre o fato da tarefa ser de fácil compreensão e de relevância acadêmica. Esse questionário inicial de sondagem foi reduzido a quatro breves questões avaliadas em escalas Likert de 4 pontos já relatadas no item "Instrumentos". Não houve diferença significativa $[t(508)=1,34$; $p>0,05 ; d=0,12]$ entre as médias das respostas logicamente válidas dos silogismos no Estudo

Tabela 1

Estatísticas Descritivas por Sexo e Total de Participantes nas Tarefas de Silogismos nas Três Condições de Conteúdos Valorativos

\begin{tabular}{lccccccccccccc}
\hline & \multicolumn{3}{c}{ Neutro } & \multicolumn{1}{c}{ Apreciativo } & \multicolumn{2}{c}{ Depreciativo } & \multicolumn{2}{c}{ Total } \\
& $N$ & $M$ & $S D$ & $N$ & $M$ & $S D$ & $N$ & $M$ & $S D$ & $N$ & $M$ & $S D$ & $I 95$ \\
\hline Feminino & 107 & 6,46 & 1,35 & 106 & 6,41 & 1,44 & 105 & 5,81 & 1,53 & 318 & 6,23 & 1,46 & $6,07-6,39$ \\
Masculino & 53 & 6,76 & 1,28 & 55 & 6,87 & 1,17 & 54 & 6,72 & 1,34 & 162 & 6,78 & 1,26 & $6,69-6,98$ \\
Total & 160 & 6,56 & 1,33 & 161 & 6,57 & 1,37 & 159 & 6,12 & 1,52 & 480 & 6,42 & 1,42 & $6,29-6,54$ \\
\hline
\end{tabular}

Os resultados foram inicialmente analisados em uma ANOVA $3 \times 2$, sendo verificado um efeito principal de grupo $[F(2,477)=3,24$; $\left.p<0,05 ; \boldsymbol{\eta}^{2}=013\right]$ e também de sexo $[F(1,478)$ $\left.=17,31 ; p<0,001 ; \boldsymbol{\eta}^{2}=0,35\right]$, mas não houve efeito interativo entre as variáveis grupo e sexo $[F$ $\left.(2,477)=1,89 ; p>0,05 ; \boldsymbol{\eta}^{2}=0,008\right]$. Em comparações post hoc (teste de Tukey), foram obtidas diferenças significativas $(p<0,05)$ entre as médias dos grupos de conteúdos depreciativos e neutros e entre as médias dos grupos de conteúdos depreciativos e apreciativos.

Observa-se, por um lado, que o grupo com conteúdos depreciativos apresentou médias $(M=6,12 ; D P=1,52)$ de respostas válidas significativamente menores $[F(2,477)=5,29 ; p<$ $0,05]$ que as dos grupos com conteúdos neutros $(M=6,56 ; D P=1,33)$ e apreciativos $(M=6,57$; $D P=1,37)$ não havendo diferenças significativas $(p>0,05)$ entre os resultados dos grupos de
Piloto $(M=6,24 ; D P=1,5)$ e na pesquisa relatada $(M=6,42 ; D P=1,42)$.

Os resultados foram analisados através do pacote estatístico SPSS-20 e foram utilizados os testes estatísticos Análise de Variância, Testes Tukey (post hoc), Teste $T$ de Student, Teste de Cohen, Eta parcial ao Quadrado (post hoc), Qui-Quadrado e Correlação de Pearson.

\section{Resultados}

\section{Resultados Obtidos nas Tarefas com Silogismos}

Os resultados referentes aos três grupos de participantes acham-se expostos na Tabela 1. 
aplicado o teste de Cohen, considerando-se a homogeneidade da amostra, obtendo-se $d=0,64$, na comparação das médias do grupo de conteúdos depreciativos, ou seja, um efeito considerado moderadamente forte. Porém, quando comparamos o desempenho de mulheres e homens nos grupos com conteúdos neutros $(d=0,22)$ e apreciativos $(d=0,3)$, o tamanho do efeito mostra-se fraco.

No que diz respeito à influência das formas de silogismos, verificou-se a maior média de respostas válidas no "Modus Ponens" ( $M=1,81$; $D P=0,5)$ e a menor no "Modus Tollens" $(M=1,37 ; D P=0,78)$. Porém, quando são comparados os resultados nas três condições de conteúdos valorativos encontramos um efeito principal de grupo, com diferenças significativas entre as médias de respostas válidas obtidas no "Modus Ponens" $\left[F(2,477)=6,38 ; \boldsymbol{p}<0,005 ; \boldsymbol{\eta}^{2}=0,26\right]$ e na "Negação do Antecedente" $[F(2,477)=$ 4,$\left.64 ; p<0,05 ; \boldsymbol{\eta}^{2}=0,019\right]$, mas não no "Modus Tollens" $\left[F(2,477)=0,77 ; p>0,05 ; \boldsymbol{\eta}^{2}=0,03\right] \mathrm{e}$ na "Afirmação do Consequente" $[F(2,477)=$ 1,$\left.47 ; p>0,05 ; \boldsymbol{\eta}^{2}=0,06\right]$. No "Modus Ponens", com a correção de Tukey, essas diferenças foram entre as médias $(M=1,88 ; D P=0,38 ; M=1,7$; $D P=0,65$, respectivamente) de respostas válidas dos grupos de conteúdos neutros e depreciativos $(p<0,005)$ e entre as médias $(M=1,86 ; D P=0,42$; $M=1,7 ; D P=0,65$, respectivamente) dos grupos de conteúdos apreciativos e depreciativos $(p<0,05)$. Já na forma "Negação do Antecedente", essas diferenças se deram, como ocorreu no "Modus Ponens", apenas entre as médias de respostas válidas dos grupos de conteúdos neutros $(M=1,66 ; D P=0,62)$ e depreciativos $(M=1,48$; $D P=0,78)$ e as dos grupos de conteúdos apreciativos $(M=1,69 ; D P=0,58)$ e depreciativos $(M=1,48 ; D P=0,78)$.

Em relação ao sexo dos participantes, foi verificada diferença significativa entre as médias de mulheres $(M=1,53 ; D P=0,72)$ e homens $(M=1,77 ; D P=0,54)$ na 'Negação do Antecedente" $[t(478)=3,70 ; p<0,001 ; d=0,38]$ mas não nas outras formas de silogismos. Quando se comparam as médias de respostas válidas apenas das mulheres, nas três condições de conteúdos valorativos, em cada forma de silogismo, foram verificadas diferenças significativas nas médias do "Modus Ponens" $[F(2,315)=4,52 ; p<0,05$; $\left.\boldsymbol{\eta}^{2}=0,028\right]$ e nas médias da "Negação do Antecedente" $\left[F(2,315)=3,41 ; p<0,05 ; \boldsymbol{\eta}^{2}=0,021\right]$. Em contraste, os homens não apresentaram diferenças significativas entre si nessas médias $[F(2$, $159)=2,23 ; p>0,05 ; \boldsymbol{\eta}^{2}=0,027 ; F(2,159)=2,51$; $p>0,05 ; \boldsymbol{\eta}^{2}=0,031$, respectivamente].

\section{Resultados Obtidos no Questionário de Vivências Acadêmicas (QVA-r)}

Os resultados obtidos no QVA-r pelos estudantes de psicologia encontram-se na Tabela 2.

Tabela 2

Média e Desvio Padrão dos Participantes nas Dimensões e no QVA-r Total

\begin{tabular}{|c|c|c|c|c|c|c|c|c|c|c|c|}
\hline \multicolumn{12}{|c|}{ Dimensões } \\
\hline \multicolumn{2}{|c|}{ Pessoal } & \multicolumn{2}{|c|}{ Interpessoal } & \multicolumn{2}{|c|}{ Carreira } & \multicolumn{2}{|c|}{ Estudo } & \multicolumn{2}{|c|}{ Institucional } & \multicolumn{2}{|c|}{ QVA-r Total } \\
\hline$M$ & $S D$ & $M$ & $S D$ & $M$ & $S D$ & $M$ & $S D$ & $M$ & $S D$ & $M$ & $S D$ \\
\hline 3,21 & 0,56 & 3,62 & 0,70 & 3,83 & 0,56 & 3,29 & 0,68 & 3,69 & 0,65 & 3,53 & 0,42 \\
\hline
\end{tabular}

Observa-se que houve pontuação acima do escore 3, escore médio da escala, em todas as dimensões e no QVA-r total, o que sugere uma integração acadêmica satisfatória dos estudantes.

Diferenças entre os sexos nas pontuações do QVA-r são apresentadas na Tabela 3 abaixo.

Como pode-se verificar, as pontuações acima do escore 3 nas dimensões da escala e no QVA-r total sugerem integração acadêmica satisfatória dos dois sexos. As mulheres obtiveram médias significativamente mais altas do que os homens nas dimensões interpessoal $[t(478)=2,0 ; p>0,05]$, carreira $[t(478)=2,31$; $p<0,05]$ e estudo [ $t(478)=2,14 ; p<0,05]$ e mais baixa na dimensão pessoal $[t(478)=2,0 ; p<0,05]$ porém o tamanho do efeito foi pequeno nessas 
Tabela 3

Estatísticas Descritivas, Teste T e Tamanho do Efeito para os Escores Médios do Sexo nas Dimensões e em QVA-r Total

\begin{tabular}{lcccccccc}
\hline \multirow{2}{*}{ Dimensões } & \multicolumn{9}{c}{ Sexo } \\
& \multicolumn{2}{c}{ Feminino } & \multicolumn{2}{c}{ Masculino } & & & & \\
\cline { 2 - 7 } & $M$ & $D P$ & $M$ & $D P$ & $t$ & $g l$ & $p$ & $d$ \\
\hline Pessoal & 3,18 & 0,53 & 3,28 & 0,59 & 2,00 & 478 & 0,05 & 0,18 \\
Interpessoal & 3,67 & 0,69 & 3,52 & 0,71 & 2,32 & 478 & 0,02 & 0,21 \\
Carreira & 3,87 & 0,53 & 3,75 & 0,60 & 2,31 & 478 & 0,02 & 0,21 \\
Estudo & 3,34 & 0,66 & 3,20 & 0,71 & 2,14 & 478 & 0,03 & 0,20 \\
Institucional & 3,71 & 0,62 & 3,65 & 0,71 & 1,01 & 478 & 0,31 & 0,09 \\
Total do QVA-r & 3,56 & 0,40 & 3,48 & 0,44 & 1,88 & 478 & 0,06 & 0,19 \\
\hline
\end{tabular}

comparações $(d=0,21 ; d=0,21 ; d=0,2 ; d=0,18$, respectivamente). Já na dimensão institucional [ $t(478)=1,01 ; p>0,05 ; d=0,09]$ e no escore do QVA-r total $[t(478)=1,88 ; p>0,05 ; d=0,19]$ não houve diferenças significativas entre os sexos. Na dimensão pessoal, os escores médios significativamente menores das mulheres compreendem os itens relacionados à "variação de humor", ao "cansaço e sonolência", aos "momentos de angústia" e à "debilidade física" que aqui são índices negativos e que, por sua vez, foram invertidos em sua valência na análise estatística, sinalizando assim maior variação de humor, maior cansaço etc.

Não houve, nos grupos de conteúdos valorativos, diferenças significativas entre as médias nas dimensões pessoal $[F(2,477)=0,27 ; p>0,05$; $\left.\boldsymbol{\eta}^{2}=0,0001\right]$, interpessoal $[F(2,477)=0,062$; $\left.p>0,05 ; \boldsymbol{\eta}^{2}=0,0001\right]$, carreira $[F(2,477)=1,07$; $\left.p>0,05 ; \quad \boldsymbol{\eta}^{2}=0,004\right]$, estudo $[F(2,477)=0,64$; $\left.p>0,05 ; \quad \boldsymbol{\eta}^{2}=0,003\right] \quad$ e institucional $[F(2$, $\left.477)=0,029 ; \quad p>0,05 ; \boldsymbol{\eta}^{2}=0,0001\right]$, bem como não houve diferença no escore médio total do QVA-r $\left[F(2,477)=0,069 ; p>0,05 ; \boldsymbol{\eta}^{2}=0,0001\right]$. Não foram observadas correlações significativas entre as dimensões e o número de respostas válidas nas tarefas com silogismos.

\section{Resultados Obtidos nas Questões sobre a Tarefa e sobre a Vida Acadêmica}

No que concerne à experiência com a tarefa e a avaliação da dificuldade da tarefa, não houve diferenças significativas entre as médias nos três grupos de conteúdos valorativos $[F(2$, $477)=0,46 ; p>0,05 ; \boldsymbol{\eta}^{2}=0,02 ; \quad F(2,477)=2,92$; $p>0,05 ; \boldsymbol{\eta}^{2}=0,12$, respectivamente]. Na avaliação da dificuldade da tarefa, o nível de significância observado aproximou-se muito de 0,05 $(p=0,052)$, sendo que a menor média obtida nessa avaliação foi a do grupo neutro e a maior média a do grupo depreciativo. Foi verificada diferença significativa entre os sexos em relação a experiência com a tarefa $[t(478)=2,56 ; p<0,05$; $d=0,25]$ e na avaliação da dificuldade da tarefa [ $t$ $(478)=3,07 ; p<0,005 ; d=0,3]$. Os homens obtiveram médias superiores $(M=2,27 ; D P=0,99)$ às das mulheres $(M=2,03 ; D P=0,93)$ na experiência com a tarefa e inferiores $(M=1,68 ; D P=0,78)$ às das mulheres $(M=1,91 ; D P=0,77)$ quanto à avaliação da dificuldade da tarefa.

Por outro lado, na questão relativa à avaliação da aplicação dos conteúdos valorativos aos psicólogos, foi verificada diferença significativa $[t(318)=9,32 ; p<0,001 ; d=1,05]$ entre as médias de concordâncias nos grupos de conteúdos apreciativos e depreciativos $(M=2,64 ; D P=0,60$; $M=1,99 ; \quad D P=0,64$, respectivamente). Como esperado, a diferença entre as médias refletiu maior concordância com os conteúdos elogiosos do grupo de conteúdos apreciativos.

Quanto à questão sobre o envolvimento afetivo com a tarefa, não houve diferença significativa $[t(318)=0,193 ; p>0,05 ; d=0,02]$ entre as médias dos grupos de conteúdos apreciativos 
e depreciativos $(M=2,6 ; D P=0,84 ; M=2,62$; $D P=1,02$, respectivamente) e não houve diferença significativa $[t(318)=0,39 ; p>0,05 ; d=0,04]$ entre as médias dos sexos feminino e masculino $(M=2,62 ; D P=0,9 ; M=2,58 ; D P=0,98$, respectivamente). Por fim, foi verificada correlação positiva fraca entre o número de respostas válidas e a experiência com a tarefa $(N=480 ; r=0,18$; $p>0,001)$.

$\mathrm{Na}$ questão aberta que buscou obter informações adicionais sobre a vida universitária, verificou-se que a maioria $(68,3 \%)$ dos participantes não a respondeu; entre os que o fizeram, 12\% relataram insatisfação com os conteúdos das disciplinas, com o currículo e/ou com os professores e colegas, dentre outras; $8 \%$ relataram aspectos positivos como satisfação com o curso; 7,7\% relataram dificuldades pessoais ou sobrecarga de estudo e trabalho; $1,3 \%$ mencionaram não saber se queriam continuar no curso e $2,7 \%$ mencionaram outros aspectos da vida universitária.

Por fim, no que diz respeito à opinião dos participantes sobre a tarefa, em questão aberta e opcional, dos $71 \%$ que a responderam, 36,8 $\%$ consideraram-na interessante, produtiva e/ ou mencionaram o interesse pelos resultados do estudo considerando que o mesmo os levou à reflexão sobre a profissão; $10,8 \%$ destacaram a dificuldade em avaliar premissas falsas e verdadeiras, sendo que destes, 5,4\% mencionaram, direta ou indiretamente, o conflito entre a lógica e os conteúdos dos silogismos; $7,3 \%$ consideraram a tarefa fácil e/ou prazerosa; $6,7 \%$ relataram não ter compreendido adequadamente os objetivos e/ou a dificuldade inicial com a tarefa que foi logo superada; $6,1 \%$ acharam a tarefa difícil e/ou ambígua; $2,3 \%$ consideraram o texto exagerado, mas intencional e $2,1 \%$ emitiram outras opiniões. Assim, a maioria dos participantes destacou aspectos positivos da tarefa.

\section{Discussão}

No que se refere ao efeito da variável sexo no raciocínio silogístico, como referido, nos grupos de conteúdos depreciativos, as estudantes do sexo feminino mostraram-se significativamente mais sensíveis a estes conteúdos, embora tenham apresentado desempenho similar ao dos estudantes do sexo masculino nos grupos de conteúdos neutros.

Estes resultados indicam, em primeiro lugar, haver um efeito negativo dos conteúdos valorativos depreciativos relativos à formação e à atuação dos psicólogos no desempenho de tarefas lógicas envolvendo silogismos em estudantes de Psicologia, especialmente, em mulheres. Sugerem ainda que essas são mais sensíveis do que os homens aos efeitos de afirmações depreciativas relativas à sua formação e à sua futura atuação profissional, embora tenham desempenho similar ao dos homens nas tarefas com silogismos com conteúdos neutros.

Embora não se tenha encontrado diferenças significativas entre as médias dos sexos masculino e feminino nos grupos de conteúdos apreciativos, a proximidade com o índice de significância $(p=0,053)$, mesmo, como visto, com o tamanho do efeito para comparação considerado pequeno $(d=0,3)$, sugere o interesse de futuros estudos que esclareçam se os homens, diferentemente das mulheres, poderiam beneficiar-se de conteúdos valorativos apreciativos relacionados à sua futura profissão.

Em alguns estudos que abordam diferenças entre homens e mulheres no raciocínio moral (Friesdorf, Conway, \& Gawronski, 2015), e na competência emocional (Day \& Carroll, 2004), os resultados sugerem que as mulheres mostraram-se mais empáticas aos problemas e aos personagens relatados nas tarefas, manifestando, consequentemente, maior competência emocional na avaliação de situações que envolvem conflito interpessoal. E, como se observou, na regulação de emoções, o maior uso de estratégias de ruminação em mulheres (Nolen-Hoeksema \& Jackson, 2001) tenderia a desviar a atenção para afetos negativos, o que poderia explicar, em parte, os resultados encontrados.

De todo modo, em diversos trabalhos (Blanchette \& Leese, 2011; Jung et al., 2014; Lefford, 1946), há o relato de efeitos mais acentuados de conteúdos afetivos negativos no desempenho em tarefas de raciocínio, o que corrobora os resultados aqui encontrados, em especial, no desempenho das estudantes do sexo feminino. 
Quanto ao impacto das formas de silogismos no desempenho, observa-se que para o total da amostra, o melhor desempenho se deu no "Modus Ponens", seguido da "Afirmação do Consequente" e da "Negação do Antecedente", e o pior desempenho no "Modus Tollens". Porém, quando os conteúdos valorativos são levados em consideração, verifica-se entre as mulheres, mas não entre os homens, um decréscimo significativo no desempenho nas tarefas silogísticas relativas aos "Modus Ponens" e "Negação do Antecedente", no grupo com conteúdos depreciativos.

Em relação a esses resultados, por um lado, seria de se esperar um decréscimo no desempenho na forma "Negação do Antecedente", porque esta forma de silogismo, logicamente inválida, é considerada difícil e mais passível de sofrer influência de crenças e expectativas dos indivíduos, bem como da tonalidade afetiva dos conteúdos, conforme apontam estudos na área (e.g. Blanchette \& Leese, 2011), podendo, por outro lado, parecer surpreendente o decréscimo de acertos no desempenho no "Modus Ponens", forma de silogismo considerada a mais fácil e direta. É possível que esse resultado se deva à rejeição à forte associação entre as premissas nas mulheres do grupo de conteúdos depreciativos. Como De Jong e Vroling (2014) observam, o "Modus Ponens" costuma ser afetado quando tal reação ocorre. Pode-se supor também que afirmações depreciativas sobre o curso de Psicologia e a formação dos psicólogos apresentadas de maneira mais direta, com forte associação de ideias, possam ter tido um papel neste resultado.

No que concerne aos resultados encontrados no QVA-r, a pontuação encontrada acima do escore 3, sugerindo boa integração acadêmica nos dois sexos, também foi obtida em outros estudos brasileiros (Igue et al., 2008; Lamas et al., 2014; Sarriera et al., 2012; Schleigh, 2006, entre outros), sendo que dois deles pesquisaram apenas estudantes de Psicologia (Igue et al., 2008; Sarriera et al., 2012).

A maior média de satisfação/ integração acadêmica ocorreu na dimensão carreira e a menor na dimensão pessoal, o que também foi observado nos estudos acima referidos. Aliás, a média maior de satisfação/ integração na di- mensão carreira no QVA-r em universitários é destacada nestes e em outros estudos (e.g. Granado et al., 2005), o que sugere que a confiança na escolha do curso e a perspectiva positiva na carreira sejam fatores relevantes para a integração acadêmica. De modo geral, as médias das dimensões do QVA-r observadas no presente estudo foram menores do que as encontradas nos trabalhos acima citados. Porém, no estudo aqui desenvolvido houve a participação apenas de estudantes de Psicologia de diferentes universidades públicas, em contraste com a amostra dos outros estudos.

Pesquisas sobre o tema (Chee, Pino, \& Smith, 2005; Tessema et al., 2012) apontam para maior satisfação acadêmica e com o currículo em mulheres. Por outro lado, estudos como de Schleigh (2006) com estudantes brasileiros, e o de Monteiro e Gonçalves (2011), com estudantes portugueses, encontraram maior satisfação com a formação no ensino superior em homens. Entretanto, estes estudos não se referem especificamente a estudantes de Psicologia. Nos estudos mencionados, desenvolvidos apenas com estudantes de Psicologia, as diferenças entre homens e mulheres não foi um ponto destacado. Não houve, no presente estudo, diferenças significativas entre as médias de integração acadêmica de cada uma das cinco dimensões e o escore médio total do QVA-r nos grupos de conteúdos valorativos, e também, não foram observadas correlações significativas entre as dimensões e o número de respostas válidas nas tarefas com silogismos.

A expectativa de que diferenças na esfera afetiva e/ou no engajamento nos estudos e na visão positiva de carreira, expressos no QVA-r, tais como os observados em mulheres e homens, pudessem se relacionar com o número de respostas válidas, considerando-se os três grupos de conteúdos valorativos não foi confirmada. Por outro lado, os resultados aqui encontrados parecem sugerir que a tonalidade depreciativa per se dos conteúdos relacionados ao curso e à formação profissional dos estudantes teve nítida influência no desempenho destes, em especial das mulheres, o que corrobora a ideia de que estes conteúdos podem afetar negativamente o desem- 
penho, independentemente da sua relação com os estados afetivos e/ou as vivências acadêmicas relatadas.

Nas questões relativas à tarefa, como visto, não foram encontradas diferenças significativas entre os sexos no grau de envolvimento com a tarefa e na avaliação da aplicação dos conteúdos valorativos ao curso e à profissão. Foram encontradas diferenças apenas na experiência com a tarefa, maior nos homens, e na avaliação da sua dificuldade, maior entre as mulheres. Assim, os estudantes do sexo masculino relataram maior familiaridade com silogismos e, consequentemente, acharam a tarefa mais fácil do que as estudantes do sexo feminino, apesar de esses resultados não terem tido influência no desempenho da tarefa quando se leva em consideração os grupos de conteúdos neutros, apreciativos e depreciativos.

Nas questões abertas acerca da vida universitária em geral, apesar da maioria dos participantes não ter respondido a esta questão, verificamos mais queixas e relatos de dificuldades naqueles que a responderam do que a menção à satisfação acadêmica, relatos estes que, de certo modo, se contrapõem ao escore geral do QVA-r que, por sua vez, sugere uma integração acadêmica satisfatória. Porém, alguns desses relatos de insatisfação referem-se a aspectos não contemplados no questionário como a relação com os professores e a adequação dos conteúdos das disciplinas e o currículo.

Em relação à opinião dos participantes sobre a tarefa, aspectos positivos da mesma foram destacados por aqueles que responderam à essa questão opcional. Neste contexto, foi interessante observar que uma parcela dos participantes fez menção ao conflito intencional entre a lógica e a tonalidade dos conteúdos, percebendo assim a natureza da tarefa, porém essa menção foi feita por um número não expressivo de participantes. Isso nos leva a supor que a maioria dos participantes não tenha percebido o conflito entre a validade lógica das tarefas e as crenças e expectativas profissionais que se contrapunham às depreciações feitas. Assim, apesar do desconforto relatado por um pequeno número de indivíduos, especialmente os que viram as histórias e os con- teúdos depreciativos, esse conflito parece ter se dado, na maioria dos participantes, de modo implícito, conforme sugerem os relatos de alguns participantes quando indagados sobre a sua opinião sobre a tarefa e se a mesma os deixou afetivamente mobilizados, coerentemente com resultados de estudos na área (Blanchette \& Amato, 2014; Blanchette \& Leese, 2011; Klauer \& Singmann, 2013) que, de certo modo, corroboram e ideia da prevalência de processos heurísticos, implícitos, associados ao Sistema 1 do modelo de Kahneman (2003), quando há conflito entre a lógica e a emoção. Porém, essa questão merece ser melhor esclarecida em estudos complementares que utilizem protocolos verbais ou outros instrumentos de auto relato que possam trazer maiores informações a esse respeito.

\section{Considerações Finais}

Os resultados da pesquisa aqui realizada podem trazer contribuições relevantes para a compreensão da influência dos conteúdos valorativos de enunciados de problemas no desempenho em tarefas lógicas que envolvem raciocínio dedutivo, em especial, de conteúdos relacionados à formação e à atuação profissional de estudantes universitários. Neste contexto, podem contribuir para dinamizar o debate de questões ainda não respondidas pelos estudos que investigam os efeitos da afetividade, em suas múltiplas expressões, no raciocínio dedutivo humano.

Concluí-se também que os efeitos dos conteúdos valorativos no raciocínio dedutivo parecem se dar de modo mais implícito, como costuma ocorrer com outros conteúdos afetivos e com interferência de crenças e expectativas, ou seja, esses efeitos parecem não depender da mediação explícita de estados afetivos e/ou da percepção dos indivíduos em seu desempenho em tarefas lógicas como as propostas no estudo.

Observa-se ainda, nos resultados, a influência per se desses conteúdos no raciocínio, pois os mesmos não foram potencializados pelo grau de integração acadêmica dos estudantes, isto é, por sua perspectiva de carreira, engajamento nos estudos e por diferenças na esfera pessoal, como relatados no QVA-r aplicado. 
Destaca-se também a necessidade de investigações futuras que esclareçam melhor se as diferenças encontradas entre homens e mulheres ocorrem também em outros cursos de graduação onde não há a prevalência do sexo feminino, como no curso de Psicologia. Os limites do estudo aqui realizado referem-se também aos seus desdobramentos futuros, particularmente, os referentes à amplificação da natureza da investigação realizada para outros cursos de graduação nas áreas biológicas e tecnológicas, bem como para grupos de indivíduos de outras faixas etárias e com graus de escolarização diferenciados.

Por fim, considera-se que os resultados encontrados podem ter particular relevância para o contexto acadêmico, na medida em que as situações-problema vivenciadas pelos alunos na universidade podem revestir-se de conteúdos valorativos que podem influenciar o desempenho na resolução de problemas e nos processos de raciocínio dedutivo, bem como em outros processos inferenciais humanos.

\section{Referências}

Almeida, S. L., Soares, A. P., \& Ferreira, J. A. (2001). Adaptação, rendimento edesenvolvimento dos estudantes no ensino superior: Construção do Questionário de Vivências Acadêmicas. Methodus, 3-20.

Blanchette, I. (2014). Does emotion affect reasoning? Yes, in multiple ways. In I. Blanchette (Ed.), Emotion and reasoning (pp. 1-21). New York: Psychology Press.

Blanchette, I., \& Amato, J. N. (2014). Reasoning and emotion in the body. In I. Blanchette (Ed.), Emotion and reasoning (pp. 119-133). New York: Psychology Press.

Blanchette, I., \& Caparos, S. (2013). When emotions improve reasoning: The possible roles of relevance and utility. Thinking \& Reasoning, 19(34). doi: 10.1080/13546783.2013.791642

Blanchette, I., \& Campbell, M. (2012). Reasoning about highly emotional topics: Syllogistic reasoning in a group of war veterans. Journal of Cognitive Psychology, 24, 157-164.

Blanchette, I., \& Leese, J. (2011). The effect of negative emotion on deductive reasoning: Examining the contribution of physiological arousal. Experimental Psychology, 58(3), 235-246.

Blanchette, I., Lindsay, P., \& Davies, S. (2014). Intense emotional experiences and logicality: An exploration of deductive reasoning in survivors of sexual abuse. The Psychological Record, 64(4), 859-867.

Blanchette, I., \& Richards, A. (2010). The influence of affect on higher level cognition: A review of research on interpretation, judgement, decision making and reasoning. Cognition \& Emotion, 24(4), 561-595.

Blanchette, I., Richards, A., Melnyk, L., \& Lavda, A. (2007). Reasoning about emotional contents following shocking terrorist attacks: A tale of three cities. Journal of Experimental Psychology: Applied, 13, 47-56.

Boden, M. T., \& Barenbaum, H. (2010). The bidirectional relations between affect and belief. $R e$ view of General Psychology, 14, 227-239.

Brody, L. R., \& Hall, J.A. (2008). Gender and emotion in context. In M. Lewis, J. M. Haviland-Jones, \& L. F. Barret (Eds.), Handbook of emotions (pp. 395-404). New York: The Guilford Press.

Chee, K. H., Pino, N. W., \& Smith, W. L. (2005). Gender differences in the academic ethic and academic achievement. College Student Journal, 39(3), 604-618.

Day, A. L., \& Carroll, S. A. (2004). Using an ability-based measure of emotional intelligence to predict individual performance, group performance, and group citizenship behaviors. Personality and Individual Differences, 36, 1443-1458.

De Jong, P., \& Vroling, M. (2014). Better safe than sorry: Threat-confirming reasoning bias in anxiety disorders. In I. Blanchette (Ed.), Emotion and reasoning (pp. 22-43). New York: Psychology Press.

De Neys, W. (2012). Bias and conflict: A case for logical intuitions. Perspectives on Psychological Sciences, 7, 28-38.

De Neys, W. (2014). Conflict, arousal, and logical gut feelings. In I. Blanchette (Ed.), Emotion and reasoning (pp. 22-43). New York: Psychology Press.

Eliades, M., Mansell, W., Stewart, A. J., \& Blanchette, I. (2012). An investigation of belief-bias and logicality on reasoning with emotional contents. Thinking \& Reasoning, 18(4), 461-479. 
Evans, J. S. T. (2008). Dual-Processing accounts of reasoning, judgment, and social cognition. $A n$ nual, Review of Psychology, 59, 255-278. doi: 10.1146/annurev.psycho.59.103006.093629

Friesdorf, R., Conway, P., \& Gawronski, B. (2015). Gender differences in responses to moral dilemmas: A process dissociation analysis. Personality and Social Psychology Bulletin, 41(5), 696713.

Gangemi, A., Mancini, F., \& Johnson-Laird, P. N. (2013). Models and cognitive change in psychopathology. Journal of Cognitive Psychology, 25(2), 131-138.

Granado, J. I. F. (2004). Vivência acadêmica de universitários brasileiros: Um estudo de validade e precisão do QVA-r (Dissertação de mestrado, Programa de Estudos de Pós-Graduação em Psicologia, Universidade de São Francisco, São Paulo, SP, Brasil).

Granado, J. I. F., Santos, A. A. A., L. S., Almeida, L. S., Soares, A. P., \& Guisande, M. A. (2005). Integração acadêmica de estudantes universitários: Contributos para a adaptação e validação do QVA-r no Brasil. Psicologia e Educação, $4(2), 31-41$.

Igue, E. A., Bariani, I. C. D., \& Milanesi, P. V. B. (2008). Vivência acadêmica e expectativas de universitários ingressantes e concluintes. PsicoUSF, 13(2), 155-164.

Johnson-Laird, P. N., Khemlani, S. S., \& Goodwin, G. P (2015). Logic, probability, and human reasoning. Trends in Cognitive Science, 20, 1-14.

Jung, N., Wranke, C., Hamburger, K., \& Knauff, M. (2014). How emotions affect logical reasoning: Evidence from experiments with mood-manipulated participant, spider phobics, and people with exam anxiety. Frontiers in Psychology, 5, $1-12$.

Kahneman, D. (2003). A perspective on judgment and choice: Mapping bounded rationality. American Psychologist, 58(9), 697-720.

Kahneman, D., \& Frederick, S. (2005). A model of heuristic judgment. In K. J. Holyoak \& R. G. Morrison (Eds.), The Cambridge handbook of thinking and reasoning (pp. 267-293), Cambridge, MA: Cambridge University Press.

Klauer, K. C., \& Singmann, H. (2013). Does logic feel good? Testing for intuitive detection of logicality in syllogistic reasoning. Journal of Experimental Psychology: Learning, Memory, and Cognition, 39(4), 1265-1273. doi: 10.1037/ a0030530.

Lamas, K. C. A., Ambiel, R. A. M., \& Silva, B. T. A. O. L. (2014). Vivências acadêmicas e empregabilidade de universitários de final de curso. Temas de Psicologia, 22(2), 61-72.

Lefford, J. S. (1946). The influence of emotional subject matter on logical reasoning. Journal of General Psychology, 34, 127-151.

Melton, R. J. (1995). The role of positive affect in syllogism performance. Personality \& Social Psychology Bulletin, 21, 788-794.

Monteiro, A. M., \& Gonçalves, C. M. (2011). Desenvolvimento vocacional no ensino superior: Satisfação com a formação e desempenho acadêmico. Revista Brasileira de Orientação Profissional, 12(1), 15-27.

Nicolle, A., \& Goel, V. (2014). What is the role of ventromedial prefrontal cortex in emotional influences on reason? In I. Blanchette (Ed.), Emotion and reasoning (pp. 154-173). New York: Psychology Press.

Nolen-Hoeksema, S., \& Jackson, B. (2001). Mediators of gender differences in rumination. Psychology of Women Quarterly, 25, 37-47.

Santos, A. A. A., Noronha, A. P. P., Amaro, C. B., \& Villar, J. (2005). Questionário de vivência acadêmica: Estudo de consistência interna do instrumento no contexto brasileiro. In M. C. R. A., Joly, A. A. A. Santos, \& F. F. Sisto (Eds.), Questões do cotidiano universitário (pp. 159178). São Paulo, SP: Casa do Psicólogo.

Sarriera, J. C., Paradiso, A. C., Schutz, F. S., \& Howes, G. P. (2012). Estudo comparativo da integração ao contexto universitário entre estudantes de diferentes instituições. Revista Brasileira de Orientação Profissional, 13(2), 163-172.

Schleigh, A. L. R. (2006). Integração à educação superior e satisfação acadêmica de estudantes ingressantes e concluintes: Um estudo sobre relações (Dissertação de mestrado, Universidade Estadual de Campinas, SP, Brasil).

Schleigh, A. L. R., Polydoro, S. A., \& Santos, A. A. A. (2006). Escala de satisfação com a experiência acadêmica de estudantes do ensino superior. Avaliação Psicológica, 5(1), 11-20.

Soares, A. B., Francischetto, V., Dutra, B. M., Miranda, J. M., Nogueira, C. C. C., Leme, V. R., ...Almeida, L. S. (2014). O impacto das expecta- 
tivas na adaptação acadêmica dos estudantes no Ensino Superior. Psico-USF, 19(1), 49-60.

Stanovich, K. E., \& West, R. (2002). Individual differences in reasoning: Implications for the rationality debate? In T. Gilovich, D. Griffin, \& D. Kahneman (Eds.), Heuristics \& biases: The psychology of intuitive judgment (pp. 421-440). New York: Cambridge University Press.

Tessema, M. T., Ready, K., \& Yu, W. (2012) Factors affecting college students' satisfaction with major curriculum: Evidence from nine years of data. International Journal of Humanities and Social Science, 2(2), 34-44.
Tversky, A., \& Kahneman, D. (1983). Extensional versus intuitive reasoning: The conjunction fallacy in probability judgment. Psychological Review, 90(4), 293-315.
Recebido: 05/10/2016

$1^{a}$ revisão: $16 / 05 / 2017$

Aceite final: 18/05/2017 